\title{
High-Quality Genome Assembly and Annotation Resource of Botryosphaeria dothidea Strain BDLA16-7, Causing Trunk Canker Disease on Chinese Hickory
}

\author{
Jiandong Bao, ${ }^{1,2}$ Qianqian Wu, ${ }^{1}$ Jianqin Huang, ${ }^{3}$ and Chuan-Qing Zhang ${ }^{1, \dagger}$ \\ ${ }^{1}$ Department of Plant Pathology, Zhejiang Agriculture and Forestry University, Hangzhou 311300, China \\ ${ }^{2}$ Fujian University Key Laboratory for Plant-Microbe Interaction, College of Life Sciences, Fujian \\ Agriculture and Forestry University, Fuzhou, 350002, China \\ ${ }^{3}$ State Key Laboratory of Subtropical Silviculture, Zhejiang Agriculture and Forestry University, Hangzhou, \\ 311300, China
}

\section{Genome Announcement}

Botryosphaeria dothidea is a latent pathogen with global importance to woody plant health, which causes serious tree trunk cankers on Chinese hickory. To date, only one Illumina short-read-based genome assembly of strain CK16 is available for host Chinese hickory. To address this problem, we report a near telomere-to-telomere genome assembly of strain BDLA16-7 using Oxford Nanopore Technologies (ONT) sequencing. The almost chromosomal-level and well-annotated genome assembly will provide a valuable genetic resource for understanding of the infection mechanisms of $B$. dothidea in future.

Chinese hickory (Carya cathayensis Sarg.) is an endemic subtropical tree species of commercial importance, which is known for its distinctive fragrance and the high nutritional value of its nuts. However, nearly $90 \%$ of the Chinese hickory trees have been severely threatened by trunk cankers caused by $B$. dothidea (Zhang and Xu 2011). This disease occurs during late March to September with obvious latent infections, for which the pathogen primarily overwinters in the diseased trunks (Dai et al. 2017; Wang and Zhang 2019). Canker symptoms generally occur on trunks of hickory less than $2 \mathrm{~m}$ above the ground, and few lesions are observed on other parts such as twigs, branches, and nuts. $B$. dothidea is a worldwide fungal pathogen with global importance: damaging woody plants; infecting through wounds; breaching the outer bark and colonizing the phloem, vascular cambium, and xylem; and causing the weakening and decay of the woody hosts (Marsberg et al. 2017; Slippers et al. 2007).

Currently, the genome sequences of six $B$. dothidea strains with host plants including apple (Liu et al. 2016; Wang et al. 2018; Yu et al. 2021), pear (Hu et al. 2019), kiwifruit (Liang et al. 2021), and Chinese hickory (Rao et al. 2021) are publicly available in the NCBI Assembly Database. The assembly sizes of the six strains were similar, ranging from 44.17 to $51.76 \mathrm{Mb}$ (Table 1). Of these strains, only one stain, named CK16, was an agent for trunk canker disease on Chinese hickory (Rao et al. 2021). The genome assembly of CK16 was assembled based on Illumina short-read sequencing technology, and has the highest contig numbers among these strains.

Here, we isolated $B$. dothidea strain BDLA16-7 from the trunk of a Chinese 'Linan' hickory tree with canker disease in Linan, Zhejiang Province, China. Its colony was initially pale white on potato dextrose agar (PDA) plates, then became black. The pycnidia were typically

${ }^{\dagger}$ Corresponding author: C. Q. Zhang; cqzhang@zafu.edu.cn

J. Bao and Q. Wu contributed equally to this work.

The author(s) declare no conflict of interest.

\section{Funding}

This work was supported by the grants from the Key Research and Development Project of Zhejiang Province, China (2020C02005), and Natural Science Foundation of Fujian Province, China (2019J01385).

\section{Keywords}

Botryosphaeria dothidea, Chinese hickory, genome, trunk canker disease 
Table 1. Genome features of the Botryosphaeria dothidea strains ${ }^{a}$

\begin{tabular}{|c|c|c|c|c|c|c|c|}
\hline Features & BDLA16-7 & CK16 & sdau11-99 & LW030101 & PG45 & LW-Hubei & PTZ1 \\
\hline Host & Chinese hickory & Chinese hickory & Apple & Apple & Apple & Pear & Kiwifruit \\
\hline Sequencing technology & ONT + Illumina & Illumina & PB + Illum & Illumina & Illumina & PB + Illum & PB + Illum \\
\hline Reference (PubMed ID) & This study & 33761770 & 33258431 & 27789635 & 30622881 & 32647612 & Liang et al. 2021 \\
\hline Assembly accession ${ }^{\mathrm{b}}$ & GWHBEBO00000000 & ASM1515935 & ASM1150312 & ASM171744 & ASM401626 & ASM1106463 & ASM1334390 \\
\hline Assembly size (Mb) & 46.05 & 44.39 & 51.76 & 47.39 & 44.17 & 46.35 & 44.45 \\
\hline Contig number & 15 & 1,303 & 88 & 1,251 & 423 & 68 & 28 \\
\hline Contig $\mathrm{N}_{50}(\mathrm{Mb})$ & 3.87 & 0.52 & 4.04 & 0.21 & 0.35 & 2.72 & 2.68 \\
\hline Contig $L_{50}$ & 5 & 26 & 6 & 65 & 37 & 7 & 7 \\
\hline Average contig length $(\mathrm{Mb})$ & 3.07 & 0.03 & 0.59 & 0.04 & 0.11 & 0.95 & 1.59 \\
\hline Maximum contig length $(\mathrm{Mb})$ & 6.19 & 1.48 & 6.15 & 1.26 & 1.15 & 4.86 & 3.62 \\
\hline GC content $(\%)$ & 54.45 & 54.60 & 52.93 & 53.09 & 54.60 & 54.30 & 54.59 \\
\hline Repeat sequence $(\%)^{c}$ & 7.96 & 5.29 & 13.96 & 12.58 & 5.64 & 9.40 & 6.68 \\
\hline BUSCO completeness (\%) ${ }^{d}$ & 99.00 & 97.83 & 97.13 & 97.83 & 97.89 & 97.71 & 97.82 \\
\hline Protein-coding genes & 12,812 & 13,856 & 14,118 & 10,411 & 15,661 & 14,091 & 10,415 \\
\hline BDLA16-7 unique genes & - & 261 & 1,745 & 1,539 & 194 & 357 & 246 \\
\hline
\end{tabular}

a Abbreviations: ONT = Oxford Nanopore Technologies, PB + Illum = PacBio + Illumina, and BUSCO = benchmarking universal single-copy orthologs.

${ }^{b}$ Assembly of BDLA16-7 is available at the Genome Warehouse (GWH) (http://ngdc.cncb.ac.cn/gwh/) while other assembles are available at the NCBI Assembly database.

${ }^{c}$ Repeats were conducted by RepeatMasker V4.1.2 with the de novo repeat library generated from assembly of BDLA16-7.

d The completeness of genome assemblies were assessed by BUSCO v5.1.2 at the Ascomycota level $(n=1,706)$.

solitary, gray-black, globular, and covered by mycelia. Its genomic DNA and messenger RNA were extracted from 10-day-old mycelium cultivated on PDA media. Its genome and transcriptome were sequenced by the ONT PromethION sequencing platform and Illumina HiSeq4000 sequencing platform, respectively, at Biomarker Technologies Co., Ltd. (Beijing, China). Finally, we obtained 5.89-Gb ONT long reads (approximately 108x, $\mathrm{N}_{50} 20.41 \mathrm{~kb}$ ) for genome assembling and 7.06-Gb Illumina short reads (approximately 156x, $2 \times 150 \mathrm{bp}$ ) for genome assembly polishing and gene annotation (Table 1).

The de novo genome assembler suit NextDenovo v2.4.0 and NextPolish v1.3.1 (both developed by NextOmics; https://github.com/Nextomics) were employed to generate a genome assembly of high accuracy and continuity. First, a draft genome assembly was assembled by NextDenovo using ONT long reads (seed reads $\geq 29,488 \mathrm{bp}$ ); then, it was polished by NextPolish using both ONT long reads and Illumina short reads. Finally, we obtained a $46.05-\mathrm{Mb}$ genome assembly (GC content $54.45 \%$ ) consisting of 15 contigs with a contig $\mathrm{N}_{50}$ of $3.87 \mathrm{Mb}\left(\mathrm{L}_{50}=5\right)$. The average contig length was $3.07 \mathrm{Mb}$ and the maximum contig length was $6.19 \mathrm{Mb}$ (Table 1). Compared with the previously reported genome assembly of strain CK16, genome assembly of BDLA16-7 was a little bigger in size (46.05 versus $44.39 \mathrm{Mb}$ ), reduced $98.85 \%$ (15 versus 1,302 ) in number of contigs, and, thus, increased approximately 100 -fold in average contig length ( 3.07 versus $0.03 \mathrm{Mb}$ ), approximately 7 -fold (3.87 versus $0.52 \mathrm{Mb}$ ) in contig $\mathrm{N}_{50}$, and approximately-4 fold (6.19 versus $1.48 \mathrm{Mb}$ ) in the maximum contig length (Table 1). Compared with the genome assemblies of other host strains, the genome assembly of BDLA16-7 had the minimum contig number and the largest contig $\mathrm{N}_{50}$, as well as the maximum contig length (Table 1).

The completeness of genome assembly BDLA16-7 was assessed by benchmarking universal single-copy orthologs (BUSCO v5.12; https://busco.ezlab.org/) at Fungi and Ascomycota levels. It contained 753 (99.34\%) complete (749 single and 4 duplicated), 3 fragmented, and 2 missing orthologs at the Fungi level $(n=758)$, and 1,689 (99.00\%) complete (1,678 single and 11 duplicated), 4 fragmented, and 13 missing orthologs at Ascomycota level $(n=1,706)$ (Fig. 1; Table 1). Compared with the BUSCO assessment results of other reported genome assemblies at the Ascomycota level, the completeness of genome assembly BDLA16-7 was the best one (Table 1). Also, the genome assembly completeness was valued by telomere repeats $\left(5^{\prime}\right.$ TTAGGG-3' and $5^{\prime}$-CCCTAA- $3^{\prime}$ ) at the start and end of contigs. Of 15 contigs, 8 and 6 contigs were found at the start or end, respectively, with telomere repeats, and 3 contigs had both ends with telomere repeats, which indicated that these contigs were reached at the telomere-to-telomere (Miga et al. 2020) chromosomal level.

Repeats were masked before gene prediction by RepeatMasker v4.1.2 (http://www. repeatmasker.org/) with a de novo repeat library generated by RepeatModeler v2.01 (http://www.repeatmasker.org/RepeatModeler). In total, $7.96 \%$ of repeats (mainly including approximately $3 \%$ long terminal repeat and approximately $2 \%$ long interspersed retrotransposable 


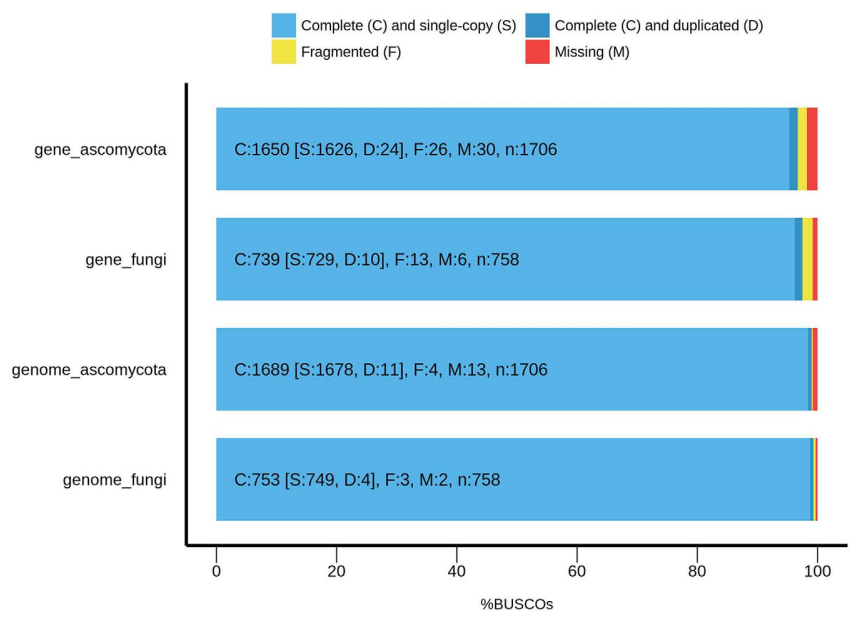

Fig. 1. Completeness of genome assembly and predicted genes were assessed by benchmarking universal single-copy orthologs (BUSCO v5.1.2) at the Fungi and Ascomycota levels.

element) were identified in the genome assembly of BDLA16-7. It is a little higher than repeats of strains CK16 (5.29\%), PG45 (5.64\%), and PTZ1 (6.68\%) but lower than repeats of strains sdau11-99 (13.96\%), LW030101 (12.58\%), and LW-Hubei (9.40\%) (Table 1). The repeat-masked genome assembly of BDLA16-7 was used for gene prediction, and identified 12,812 proteincoding genes by BRAKER2 (Bruna et al. 2021), which integrated ab initio gene prediction from AUGUSTUS v3.4.0 (Stanke et al. 2008), as well as evidence from RNA-sequencing data and fungal homologous proteins (fungi_odb10) (https://busco-data.ezlab.org/v5/data/lineages/). Compared with other strains, the number of protein-coding genes of BDLA16-7 was in the middle $(12,815$ versus approximately 10,411 to 15,661$)$, which may have been caused by different gene prediction methods. Using BLASTn search against other genome assemblies, the number of isolate-specific genes in BDLA16-7 ranged from 194 to 1,745 (Table 1). The BUSCO gene completeness assessment showed that the predicted gene set of BDLA16-7 included 739 (97.50\%) and 1,650 (96.72\%) complete orthologs at the Fungi and Ascomycota levels, respectively (Fig. 1).

The gene functional annotation was conducted by InterProScan v5.51-85.0 (https:// github.com/ebi-pf-team/interproscan) and eggNOG-mapper v2 (http://eggnog-mapper.embl. de/). In total, 10,272 (80.16\%) genes were assigned a annotation from either Pfam $(9,231)$, gene ontology $(4,027)$, Kyoto Encyclopedia of Genes and Genomes $(4,741)$, or eukaryotic orthologous groups $(9,998)$. We identified a set of pathogenicity-related genes, including 3,642 pathogen-host interaction genes (PHI-base v4.12; http://www.phi-base.org/), 250 carbohydrate active enzymes (dbCAN2; https://bcb.unl.edu/dbCAN2/), and 252 cytochrome P450 enzymes. Furthermore, 752 putative secreted proteins were identified by SignalP v5.0 (Almagro Armenteros et al. 2019) and TMHMM v2.0 (http://www.cbs.dtu.dk/services/ $\mathrm{TMHMM} /$ ), following our previous method (with signal peptide but without any transmembrane domain in mature protein) (Bao et al. 2017).

Fungal secondary metabolites such as fumonisins and deoxynivalenol were toxic to plants and, thus, played an important role in fungal infection and plant disease development (Diamond et al. 2013; lqbal et al. 2021). The genes related to products of any secondary metabolite were fused as secondary metabolite biosynthesis gene clusters (SMBGCs) (Keller 2019). In total, 63 SMBGCs, including 18 nonribosomal peptide synthetases (NRPSs), 13 NPRS-like, 11 terpenes, 19 type I polyketide synthases, and $2 \beta$-lactones were identified by the fungal version of antiSMASH v5.2.0 (Blin et al. 2019).

The whole-genome sequence data reported in this article have been deposited in the Genome Warehouse (GWH) (https://ngdc.cncb.ac.cn/gwh/) in the National Genomics Data Center, China National Center for Bioinformation (CNCB-NGDC Members and Partners 2021), under accession number GWHBEBO00000000. The raw sequence reads also are publicly accessible at Genome Sequence Archive (https://ngdc.cncb.ac.cn/gsa/) in CNCBNGDC under accession number CRA004612 (BioProject PRJCA005744). 


\section{Literature Cited}

Almagro Armenteros, J. J., Tsirigos, K. D., Sønderby, C. K., Petersen, T. N., Winther, O., Brunak, S., von Heijne, G., and Nielsen, H. 2019. SignalP 5.0 improves signal peptide predictions using deep neural networks. Nat. Biotechnol. 37:420-423.

Bao, J., Chen, M., Zhong, Z., Tang, W., Lin, L., Zhang, X., Jiang, H., Zhang, D., Miao, C., Tang, H., Zhang, J., Lu, G., Ming, R., Norvienyeku, J., Wang, B., and Wang, Z. 2017. PacBio sequencing reveals transposable elements as a key contributor to genomic plasticity and virulence variation in Magnaporthe oryzae. Mol. Plant 10:1465-1468.

Blin, K., Shaw, S., Steinke, K., Villebro, R., Ziemert, N., Lee, S. Y., Medema, M. H., and Weber, T. 2019. AntiSMASH 5.0: Updates to the secondary metabolite genome mining pipeline. Nucleic Acids Res. 47:W81-W87.

Buina, T., Hoff, K. J., Lomsadze, A., Stanke, M., and Borodovsky, M. 2021. BRAKER2: Automatic eukaryotic genome annotation with GeneMark-EP+ and AUGUSTUS supported by a protein database. NAR Genomics Bioinf. 3:Iqaa108.

CNCB-NGDC Members and Partners. 2021. Database resources of the national genomics data center, China national center for bioinformation in 2021. Nucleic Acids Res. 49:D18-D28.

Dai, D. J., Wang, H. D., Wang, Y. P., and Zhang, C. Q. 2017. Management of Chinese hickory (Carya cathayensis) trunk canker through effective fungicide application programs and baseline sensitivity of Botryosphaeria dothidea to trifloxystrobin. Australas. Plant Pathol. 46:75-82.

Diamond, M., Reape, T. J., Rocha, O., Doyle, S. M., Kacprzyk, J., Doohan, F. M., and McCabe, P. F. 2013. The Fusarium mycotoxin deoxynivalenol can inhibit plant apoptosis-like programmed cell death. PLoS One 8:e69542.

Hu, W., Luo, H., Yang, Y., Wang, Q., Hong, N., Wang, G., Wang, A., and Wang, L. 2019. Comprehensive analysis of full genome sequence and Bd-milRNA/target mRNAs to discover the mechanism of hypovirulence in Botryosphaeria dothidea strains on pear infection with BdCV1 and BdPV1. IMA Fungus 10:3-28.

Iqbal, N., Czékus, Z., Poór, P., and Ördög, A. 2021. Plant defence mechanisms against mycotoxin Fumonisin B1. Chem. Biol. Interact. 343:109494.

Keller, N. P. 2019. Fungal secondary metabolism: Regulation, function and drug discovery. Nat. Rev. Microbiol. 17:167-180.

Liang, K., Lan, J., Wang, B., Liu, Y., Lu, Q., and Liu, P. 2021. High-quality genome resource of the pathogen of Botryosphaeria dothidea causing kiwifruit soft rot. PhytoFrontiers. 1:123-125.

Liu, Z., Lian, S., Li, B., Lu, H., Dong, X., and Wang, C. 2016. Draft genome sequence of Botryosphaeria dothidea, the pathogen of apple ring rot. Genome Announce. 4:e01142-16.
Marsberg, A., Kemler, M., Jami, F., Nagel, J. H., Postma-Smidt, A., Naidoo, S., Wingfield, M. J., Crous, P. W., Spatafora, J. W., Hesse, C. N., Robbertse, B., and Slippers, B. 2017. Botryosphaeria dothidea: A latent pathogen of global importance to woody plant health. Mol. Plant Pathol. 18:477-488.

Miga, K. H., Koren, S., Rhie, A., Vollger, M. R., Gershman, A., Bzikadze, A., Brooks, S., Howe, E., Porubsky, D., Logsdon, G. A., Schneider, V. A., Potapova, T., Wood, J., Chow, W., Armstrong, J., Fredrickson, J., Pak, E., Tigyi, K., Kremitzki, M., Markovic, C., Maduro, V., Dutra, A., Bouffard, G. G., Chang, A. M., Hansen, N. F., Wilfert, A. B., Thibaud-Nissen, F., Schmitt, A. D., Belton, J.-M., Selvaraj, S., Dennis, M. Y., Soto, D. C., Sahasrabudhe, R., Kaya, G., Quick, J., Loman, N. J., Holmes, N., Loose, M., Surti, U., ana Risques, R., Lindsay, T. A. G., Fulton, R., Hall, I., Paten, B., Howe, K., Timp, W., Young, A., Mullikin, J. C., Pevzner, P. A., Gerton, J. L., Sullivan, B. A., Eichler, E. E., and Phillippy, A. M. 2020. Telomere-to-telomere assembly of a complete human $X$ chromosome. Nature 585:79-84.

Rao, Y., Mei, L., Zhang, L., Jiang, H., Ma, L., and Wang, Y. 2021. Genome sequence resource of Botryosphaeria dothidea CK16, a fungal pathogen causing Chinese hickory trunk canker disease. Plant Dis. 105:3282-3284.

Slippers, B., Smit, W. A., Crous, P. W., Coutinho, T. A., Wingfield, B. D., and Wingfield, M. J. 2007. Taxonomy, phylogeny and identification of Botryosphaeriaceae associated with pome and stone fruit trees in South Africa and other regions of the world. Plant Pathol. 56:128-139.

Stanke, M., Diekhans, M., Baertsch, R., and Haussler, D. 2008. Using native and syntenically mapped cDNA alignments to improve de novo gene finding. Bioinformatics 24:637-644.

Wang, B., Liang, X., Gleason, M. L., Zhang, R., and Sun, G. 2018. Comparative genomics of Botryosphaeria dothidea and $B$. kuwatsukai, causal agents of apple ring rot, reveals both species expansion of pathogenicity related genes and variations in virulence gene content during speciation. IMA Fungus 9: 243-257.

Wang, Q. W., and Zhang, C. Q. 2019. q-LAMP assays for the detection of Botryosphaeria dothidea causing Chinese hickory canker in trunk, water, and air samples. Plant Dis. 103:3142-3149.

Yu, C., Diao, Y., Lu, Q., Zhao, J., Cui, S., Peng, C., He, B., Liang, Y., and Liu, H. 2021. Genome assembly and annotation of Botryosphaeria dothidea sdau11-99, a latent pathogen of apple fruit ring rot in China. Plant Dis. 105: 1555-1557.

Zhang, C. Q., and Xu, B. C. 2011. First report of canker on Chinese hickory (Carya cathayensis) caused by Botryosphaeria dothidea in China. Plant Dis. 95:1319. 